\title{
The effect of preoperative chemotherapy treatment in surgically treated intrahepatic cholangiocarcinoma patients-A multi-institutional analysis
}

\author{
Stefan Buettner ${ }^{1,2}$ | Bas Groot Koerkamp ${ }^{2}$ | Aslam Ejaz ${ }^{1}$ | \\ Florian E. Buisman ${ }^{2}$ | Yuhree Kim ${ }^{1}$ | Georgios Antonios Margonis ${ }^{1}$ \\ Sorin Alexandrescu ${ }^{3}$ | Hugo P. Marques ${ }^{4}$ | Jorge Lamelas ${ }^{4}$ | Luca Aldrighetti ${ }^{5}$ | \\ T. Clark Gamblin ${ }^{6}$ | Shishir K. Maithel ${ }^{7}$ | Carlo Pulitano ${ }^{8}$ | Todd W. Bauer $^{9}$ | \\ Feng Shen ${ }^{10}$ | George A. Poultsides ${ }^{11}$ | J. Wallis Marsh ${ }^{12}$ | Jan N.M. IJzermans ${ }^{2}$ | \\ Timothy M. Pawlik ${ }^{1}$
}

${ }^{1}$ Department of Surgery, Johns Hopkins

Hospital, Baltimore, Maryland

${ }^{2}$ Department of Surgery, Erasmus Medical Center, Rotterdam, the Netherlands

${ }^{3}$ Fundeni Clinical Institute, Bucharest, Romania

${ }^{4}$ Curry Cabral Hospital, Lisbon, Portugal

${ }^{5}$ Ospedale San Raffaele, Milan, Italy

${ }^{6}$ Medical College of Wisconsin, Milwaukee, Wisconsin

7 Emory University, Atlanta, Georgia

8 University of Sydney, Sydney, Australia

9 University of Virginia, Charlottesville, Virginia

${ }^{10}$ Eastern Hepatobiliary Surgery Hospital,

Shanghai, China

${ }^{11}$ Stanford University, Stanford, California

12 University of Pittsburgh Medical Center,

Pittsburgh, Pennsylvania

\section{Correspondence}

Timothy M. Pawlik, MD, MPH, PhD, FACS,

Professor and Chair, Department of Surgery,

The Urban Meyer III and Shelley Meyer Chair for Cancer Research, The Ohio State University Wexner Medical Center, 395 W. 12th Avenue,

Suite 670, Columbus, $\mathrm{OH} 43210$.

Email: tim.pawlik@osumc.edu
INTRODUCTION: While preoperative chemotherapy $(\mathrm{pCT})$ is utilized in many intra-abdominal cancers, the use of pCT among patients with intrahepatic cholangiocarcinoma (ICC) remains ill defined. As such, the objective of the current study was to examine the impact of pCT among patients undergoing curative-intent resection for ICC.

METHODS: Patients who underwent hepatectomy for ICC were identified from a multiinstitutional international cohort. The association between PCT with peri-operative and longterm clinical outcomes was assessed.

RESULTS: Of the 1057 patients who were identified and met the inclusion criteria, 62 patients (5.9\%) received $\mathrm{pCT}$. These patients were noticed to have more advanced disease. Median OS (pCT:46.9 months vs no pCT:37.4 months; $P=0.900$ ) and DFS ( 29.1 months; $P=0.909$ ) were similar between the two groups. In a subgroup analysis of propensity-score matched patients, there was longer OS ( months) and DFS (pCT:34.1 months vs no pCT:14.0 months); however this did not reach statistical significance (both $P>0.05$ ).

CONCLUSION: In conclusion, $\mathrm{pCT}$ utilization among patients with ICC was higher among patients with more advanced disease. Short-term post-operative outcomes were not affected by pCT use and receipt of $\mathrm{pCT}$ resulted in equivalent OS and DFS following curative-intent resection.

KEYWORDS

intrahepatic cholangiocarcinoma, preoperative chemotherapy, prognostic factors, risk factors, survival

\section{1 | INTRODUCTION}

Intrahepatic cholangiocarcinoma (ICC) is the second most common primary liver malignancy, accounting for $3 \%$ of all cases of gastrointestinal cancer. ${ }^{1,2}$ ICC makes up about $5-10 \%$ of all cholangiocarcinomas and originates from bile ducts within the liver parenchyma. ${ }^{2,3}$
Histologically, the majority of advanced ICC tumors are adenocarcinoma, which are typically treated with a combination of cytotoxic nucleoside analogs and platins., ${ }^{4,5}$ When feasible, complete surgical resection of ICC remains the only possible option for cure with an estimated median survival ranging from 27 to 36 months. ${ }^{6-9}$ However, only a minority of patients with ICC present with surgically resectable 
disease at the time of diagnosis. Even with complete surgical resection, recurrence can be as high as 50\% within 24 months of surgical resection. ${ }^{10}$ In addition, nearly one in five patients undergoing curative-intent resection are left with microscopic disease following surgery. ${ }^{11}$ As such, there has been interest in using preoperative chemotherapy $(\mathrm{pCT})$ to improve patient selection, increase the incidence of margin negative surgical resection and potentially improve disease-free (DFS) and overall survival (OS).

Preoperative chemotherapy is utilized in many intra-abdominal cancers to reduce local disease burden and the incidence of micrometastatic disease prior to surgical resection. In patients with perihilar cholangiocarcinoma ( $\mathrm{PHC}$ ), recent trials have shown that $\mathrm{PCT}$ may be effective in increasing DFS. ${ }^{12}$ Furthermore, in patients with PHC, pCT can down-size locally advanced tumors in order to help facilitate surgical resection. ${ }^{12}$ Despite this, the use of $\mathrm{PCT}$ among patients with ICC has not been well-studied. ${ }^{5,12}$ As such, the objective of the current study was to determine the impact of pCT on OS and DFS in a large, multi-institutional international cohort of patients who underwent curative-intent resection for ICC. Furthermore, we sought to characterize current practice patterns regarding the use of $\mathrm{pCT}$ among patients undergoing curative-intent resection for ICC.

\section{2 | METHODS}

All patients undergoing curative-intent resection for ICC between January 1, 1990, and July 1, 2016 at one of 12 participating major hepatobiliary institutions in the United States, Asia, Oceania, and Europe were identified (Johns Hopkins University, Baltimore, Maryland; Emory University, Atlanta, Georgia; Stanford University Medical Center, Stanford, California; University of Virginia Health System, Charlottesville, Virginia; Fundeni Clinical Institute, Bucharest, Romania; Beaujon Hospital, Clichy, France; Curry Cabral Hospital, Lisbon, Portugal; Eastern Hepatobiliary Surgery Hospital, Shanghai, China; Ottowa General Hospital, Ottowa, Canada; Royal Prince Alfred Hospital, Sydney, Australia; San Raffaele Hospital, Milan, Italy; Erasmus University Medical Centre Rotterdam, Rotterdam, the Netherlands). Sociodemographic and clinicopathologic data were collected and include age, sex, and race, tumor size, AJCC stage, histologic grade, presence of nodal metastases, final resection margin and the presence of vascular and/or perineural invasion.

A minor hepatectomy was defined as a hepatic resection of less than 3 Couinaud segments. Suspected lymph node metastases on preoperative scans were considered suspicious preoperative lymph nodes, while only pathologically proven metastases were considered proven metastases. Patients with suspected lymph nodes preoperatively, and confirmed lymph node metastases during pathological examination of the resection specimen, were considered to have lymph node disease preoperatively and postoperatively, respectively. Patients with suspected lymph node disease preoperatively, but no evidence in the resection specimen, were considered to only have lymph node metastases preoperatively.

The American Joint Committee on Cancer (AJCC) 7th edition staging was used to stratify patients by extent of disease. ${ }^{13}$ Margin status was categorized as R0 for a negative margin, R1 when the margin was microscopically positive and R2 when the margin was macroscopically positive. Only patients undergoing a curative intent surgery for histologically confirmed ICC were included in the final study population; patients who did not undergo resection were excluded. Patients who underwent transplantation were also excluded. The respective institutional review boards of each participating institution approved this study.

\section{1 | Statistical analysis}

Categorical variables were described as whole numbers and percentages while continuous variables were reported as medians with interquartile (IQR) range. Percentages for each variable were calculated based on available data, excluding missing values. Univariable comparison of categorical variables was performed using the Pearson chi-square test. Univariable comparison of continuous variables was performed using the Mann-Whitney U-test. The primary outcome of the study was 5-year OS. OS was calculated as the time from the date of surgery to the date of death or date of last available follow-up; OS was estimated using the Kaplan-Meier method. DFS was calculated from the date of surgery to the date of first-known radiographically or pathologically confirmed metastasis. Logistic regression analysis was conducted in order to determine factors associated with receipt of $\mathrm{pCT}$ in a multivariable model. Based on this regression model, a propensity score was calculated to determine the likelihood of receiving $\mathrm{pCT}$. Patients were matched based on this propensity score and OS was compared between the groups. All analyses were performed using SPSS 22.0 (IBM, New York). All tests were 2 -sided and $P<0.05$ defined statistical significance.

\section{3 | RESULTS}

\section{1 | Clinical and pathologic description of patient cohort receiving $\mathrm{PCT}$}

1057 patients who underwent curative-intent resection for ICC and met the inclusion criteria were identified; 62 patients (5.9\%) received pCT (Table 1). Among the patients who received pCT, 18 (29.0\%) patients were treated with intra-arterial chemotherapy, while the remaining 44 patients (71.0\%) were treated with systemic chemotherapy. Median patient age among patients who received pCT was 60 years (IQR 52, 69) and the majority of the patients were male ( $n=37,59.7 \%$ ). Most patients had an ASA classification of II or III $(n=51,92.7 \%)$.

Based on preoperative imaging and/or biopsy, over one-third of patients had suspected or proven lymph node metastases $(n=21$, $39.6 \%$ ). We observed that patients who received systemic chemotherapy more frequently had suspected or confirmed lymph node metastases ( $n=17,44.7 \%$ ), compared to patients who received intraarterial chemotherapy $(n=4,26.7 \%)$. However, this difference did not reach statistical significance $(P=0.226)$. At the time of surgery, approximately one-half of patients underwent a major hepatectomy 
TABLE 1 Characteristics of the preoperative chemotherapy group $(n=62)$

\begin{tabular}{|c|c|}
\hline Variable & $n(\%) /$ median (IQR) \\
\hline \multicolumn{2}{|l|}{ Gender } \\
\hline Male & $37(59.7)$ \\
\hline Female & $25(40.3)$ \\
\hline Age, years & $60(52-69)$ \\
\hline \multicolumn{2}{|l|}{ Race } \\
\hline Caucasian & $49(79.0)$ \\
\hline African-American & $8(12.9)$ \\
\hline Other & $5(8.0)$ \\
\hline \multicolumn{2}{|l|}{ ASA } \\
\hline 1 & $3(5.5)$ \\
\hline II & $24(43.6)$ \\
\hline III & $27(49.1)$ \\
\hline IV & $1(1.8)$ \\
\hline BMI & $25.8(23.5-29.0)$ \\
\hline \multicolumn{2}{|l|}{ Period of treatment } \\
\hline $1990-2000$ & $3(5.3)$ \\
\hline $2001-2005$ & $4(7.0)$ \\
\hline $2006-2010$ & $20(35.1)$ \\
\hline $2011-2016$ & $30(52.6)$ \\
\hline \multicolumn{2}{|l|}{ Preoperative chemotherapy type } \\
\hline Intra-arterial therapy & $18(29.0)$ \\
\hline Systemic therapy & $44(71.0)$ \\
\hline \multicolumn{2}{|l|}{ Preoperative lymph node metastases } \\
\hline No & $32(60.4)$ \\
\hline Suspicious & $12(22.6)$ \\
\hline Proven & $9(17.0)$ \\
\hline \multicolumn{2}{|l|}{ Type of resection } \\
\hline Minor hepatectomy ( $<3$ segments) & $6(10.9)$ \\
\hline Right hepatectomy & $9(16.4)$ \\
\hline Left hepatectomy & $8(14.5)$ \\
\hline Extended right hepatectomy & $18(32.7)$ \\
\hline Extended left hepatectomy & $11(20.0)$ \\
\hline Central hepatectomy & $3(5.5)$ \\
\hline Lymphadenectomy & $39(70.9)$ \\
\hline Lymph nodes harvested & $3(1-6)$ \\
\hline Lymph node metastases & $15(24.2)$ \\
\hline Extrahepatic metastases & $8(12.9)$ \\
\hline \multicolumn{2}{|l|}{ Margin status } \\
\hline RO & $42(73.7)$ \\
\hline $\mathrm{R} 1$ & $14(24.6)$ \\
\hline $\mathrm{R} 2$ & $1(1.8)$ \\
\hline
\end{tabular}

involving more than three Couinaud segments $(n=29,52.7 \%)$. The majority of patients underwent a formal portal lymphadenectomy ( $n=39,70.9 \%$ ), with a median of 3 lymph nodes (IQR: 1,6$)$ examined. On final pathology, the majority of patients had an R0 resection $(n=42$, $73.7 \%)$. Lymph node metastasis was noted in $24.2 \%$ of patients
( $n=15)$. Twelve patients $(25.5 \%)$ who had lymph node metastases on the preoperative work-up did not have lymph node metastasis on final pathology.

\subsection{Receipt of preoperative chemotherapy}

The majority of patients who received $\mathrm{pCT}(n=50)$ were treated within the past 10 years, however the rate of $\mathrm{pCT}$ remained stable over the study period $(P=0.632)$. Several clinicopathologic features were associated with receipt of $\mathrm{pCT}$ (Table 2). Preoperatively, patients with suspected or biopsy-proven lymph nodes more likely received PCT (39.6\% vs $18.5 \%, P<0.001)$. Patients who received $\mathrm{pCT}$ were also more likely to have advanced disease compared with patients who did not receive $\mathrm{pCT}$. Specifically, patients with microvascular invasion (pCT: $n=25,48.1 \%$ vs no pCT: $n=232,24.4 \% ; P<0.001$ ) and perineural invasion (pCT: $n=15,30.6 \%$ vs no pCT: $n=137,15.6 \%$; $P=0.006$ ) more commonly received $\mathrm{pCT}$. Furthermore, based on the AJCC 7th edition staging system, patients who received $\mathrm{pCT}$ more commonly had stage III or IV disease (pCT: $n=16,55.2 \%$ vs no pCT: $n=146,24.7 ; P<0.001)$. The presence of extrahepatic disease was also associated with receipt of $\mathrm{pCT}$ (рCT: $n=8,12.9 \%$ vs no pCT: $n=32,3.2 ; P<0.001$ ). On final pathology, patients who received pCT also more often had microscopic R1 (pCT: $n=14,24.6 \%$ vs no pCT: $n=120,12.4 \%$; or macroscopic R2 (pCT: $n=1,1.8 \%$ vs no pCT: $n=4,0.4 \% ; P=0.010$ ) resections.

On multivariable analysis, after controlling for all measurable confounders, factors associated with receipt of $\mathrm{PCT}$ included major hepatic resection (OR: $3.88,95 \% \mathrm{Cl} 1.43-10.49, P=0.008$ ) and the presence of microvascular invasion (OR: $2.93,95 \% \mathrm{Cl} 1.43-6.02$, $P=0.003)$.

\section{3 | Perioperative morbidity}

Overall morbidity among all patients who underwent resection for ICC was $40.2 \%$ ( $n=420$ ) with a higher incidence of complications occurring among patients who received pCT ( $\mathrm{pCT}: n=36,59.0 \%$ vs no pCT: $n=384,39.0 \% ; P=0.002$ ); major morbidity, however, did not differ between the two groups $(P=0.568)$ (Table 3). Median length of stay (pCT: 9 days, IQR 6,15 vs no pCT: 12 days, IQR 7,17; $P=0.080$ ) and perioperative mortality within 90 days of surgery $(\mathrm{pCT}: n=1,2.2 \%$ vs no pCT: $n=35,3.9 \% ; P=0.569$ ) also did not differ between the two groups. Readmission within 30 days from surgery, however, was more common among patients who received pCT ( $\mathrm{pCT}: n=8,15.7 \%$ vs no pCT: $n=39,4.8 \% ; P=0.001)$. Post-operatively, patients in the pCT group more often received adjuvant chemotherapy $(50.8 \%$ vs $29.0 \%$, $P=0.001)$.

\subsection{Impact of preoperative chemotherapy on overall and disease-free survival}

At a median follow-up of 27.6 months, mortality occurred in 522 (49.7\%) patients. Median OS among the entire cohort was 37.4 months (95\% $\mathrm{Cl} 32.5-42.3$ months) with 1-, 3-, and 5-year OS being $78.9 \%, 51.4 \%$, and $39.2 \%$, respectively. Stratified by receipt of pCT, median OS was similar between the two groups (pCT: 46.9 months, 
TABLE 2 Comparison of disease characteristics across treatment groups

\begin{tabular}{|c|c|c|c|}
\hline Variable & No Preoperative Chemotherapy $(n=995)$ & Preoperative Chemotherapy $(n=62)$ & $P$-value \\
\hline Preoperative lymph node metastases & & & $<0.001$ \\
\hline No & $699(81.2)$ & $32(60.4)$ & \\
\hline Proven & $38(4.4)$ & $9(17.0)$ & \\
\hline Type of resection & & & $<0.001$ \\
\hline Left hepatectomy & $185(19.1)$ & $8(14.5)$ & \\
\hline Extended right hepatectomy & $110(11.4)$ & $18(32.7)$ & \\
\hline Extended left hepatectomy & $85(8.8)$ & $11(20.0)$ & \\
\hline Central hepatectomy & $18(1.9)$ & $3(5.5)$ & \\
\hline Microvascular invasion & $232(24.4)$ & $25(48.1)$ & $<0.001$ \\
\hline Perineural invasion & $137(15.6)$ & $15(30.6)$ & 0.006 \\
\hline Invasion of adjacent organs & $72(7.4)$ & $5(8.9)$ & 0.676 \\
\hline Satellite lesions & $216(22.2)$ & $17(29.8)$ & 0.181 \\
\hline Intrahepatic metastases & $69(7.1)$ & $6(10.7)$ & 0.308 \\
\hline Lymphadenectomy & $424(43.7)$ & $39(70.9)$ & $<0.001$ \\
\hline Lymph nodes harvested & $2(0-5)$ & $3(1-6)$ & 0.074 \\
\hline Lymph node metastases & $169(17.0)$ & $15(24.2)$ & 0.146 \\
\hline 1 & $282(48.0)$ & $7(24.1)$ & \\
\hline II & $160(27.2)$ & $6(20.7)$ & \\
\hline III & $22(3.7)$ & $6(20.7)$ & \\
\hline IVA & $112(19.0)$ & $8(27.6)$ & \\
\hline IVB & $12(2.0)$ & $2(6.9)$ & \\
\hline
\end{tabular}

95\%Cl 28.5-65.2 months vs no pCT: 37.4 months, 95\% Cl 32.3-42.5 months; $P=0.900$; Fig. 1). Disease recurrence occurred in 454 (43.0\%) patients. Median DFS among the entire cohort was 29.6 months $(95 \%$ Cl 17.2-42.0 months) with 1-, 3-, and 5-year DFS being 64.7\%, 46.6\%, and $44.4 \%$, respectively. Stratified by receipt of pCT, median DFS was also similar between the two groups (рСT: 34.1 months, $95 \% \mathrm{Cl}$ 2.5-65.7 months vs no pCT: 29.1 months, $95 \% \mathrm{Cl} 16.0-42.2$ months; $P=0.909$; Fig. 2).

In a subgroup analysis of propensity-score matched patients based on the factors associated with receipt of pCT $(n=100)$, there was longer OS in the pCT group (pCT: 46.9 months, $95 \% \mathrm{Cl}$ 24.3-69.4 months vs no pCT: 29.4 months, $95 \% \mathrm{Cl} 14.5-44.4$ months), however this did not reach statistical significance $(P=0.136$; Fig. 3). Similarly, there was suggestion of an improved DFS in the pCT group (pCT: 34.1 months, $95 \% \mathrm{Cl} 0-70.2$ months vs no pCT:14.0 months, $95 \% \mathrm{Cl}$ 7.0-20.9 months; $P=0.551$ ).

\section{4 | DISCUSSION}

Preoperative therapy is used in several intra-abdominal cancers to reduce local and micrometastatic tumor burden prior to complete surgical resection. Some benefits of PCT include the potential to down-size tumors to increase resectability rates among patients who are initially deemed unresectable. Furthermore, pCT can potentially improve completeness of surgical resection, as well as help select patients with a better tumor biology, thereby improving OS and DFS. In the current study, we examined a large, multi-institutional 
TABLE 3 Comparison of postoperative course and follow-up across treatment groups

\begin{tabular}{|c|c|c|c|}
\hline Variable & No preoperative chemotherapy $(n=995)$ & Preoperative chemotherapy ( $n=62$ ) & $P$-value \\
\hline Complication & $384(39.0)$ & $36(59.0)$ & 0.002 \\
\hline Clavien dindo grade & & & 0.568 \\
\hline III-V & $173(42.0)$ & $20(46.5)$ & \\
\hline Length of stay (days) & $12(7-17)$ & $9(6-15)$ & 0.080 \\
\hline Postoperative mortality & $35(3.9)$ & $1(2.2)$ & 0.569 \\
\hline \multicolumn{4}{|l|}{ Adjuvant therapy } \\
\hline Adjuvant intra-arterial therapy & $102(14.1)$ & $7(14.6)$ & 0.921 \\
\hline Adjuvant chemotherapy & $270(29.0)$ & $30(50.8)$ & 0.001 \\
\hline Adjuvant radiotherapy & $56(6.4)$ & $6(10.7)$ & 0.203 \\
\hline
\end{tabular}

international cohort of patients receiving PCT for ICC. As the use of PCT among patients with ICC has not been well-studied, this represents to our knowledge the largest study to date analyzing the impact of $\mathrm{pCT}$ among patients undergoing curative-intent resection for ICC. We noted that patients with more advanced disease were more likely to receive $\mathrm{pCT}$. Of note, the use of $\mathrm{pCT}$ did result in higher overall but not major perioperative morbidity. Furthermore, in the propensity score-matched cohort, there was a suggestion that $\mathrm{PCT}$ improved OS and DFS, however these differences did not reach statistical significance perhaps due to a small sample size.

The use of pCT has not been examined among patients with ICC in any prospective clinical trial to date. Likely due to the overall low incidence of ICC, patients with ICC are often grouped in clinical trials with other patients with biliary tract cancers. As such, the benefit of pCT in patients with ICC is ill-defined and not commonly utilized. ${ }^{2,14-16}$ In fact, in the current multi-institutional international cohort, the overall utilization of pCT was only $5.9 \%$. This is likely due to the fact that analyses from available studies have been unable to show a reproducible benefit with the use of $\mathrm{PCT}$ among patients with ICC. ${ }^{2,16}$ Among patients with pancreatic adenocarcinoma, however, $\mathrm{pCT}$ has been used in patients with locally advanced tumors to define the tumor biology. ${ }^{17}$ In the current cohort, patients with more advanced disease were more

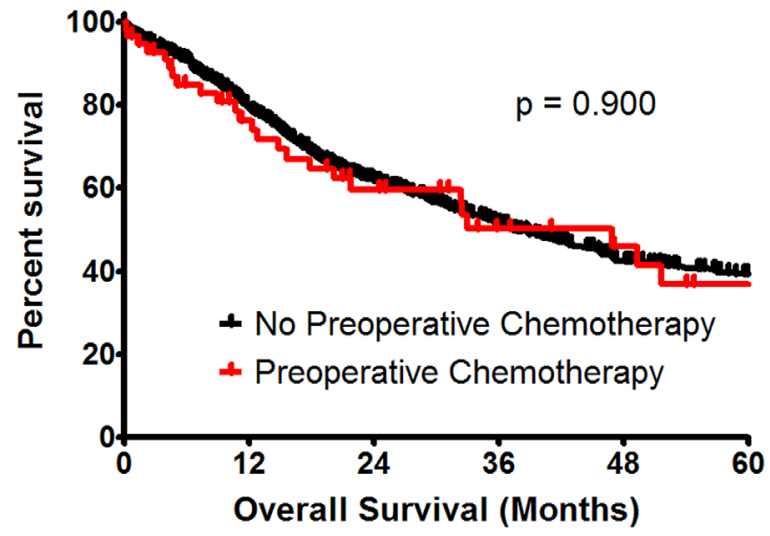

FIGURE 1 Overall survival stratified by preoperative chemotherapy $(P=0.900)$ likely to receive $\mathrm{pCT}$ - suggesting that physicians were using $\mathrm{pCT}$, in part, to help define the natural history of the disease. Specifically, patients with more preoperative suspected or biopsy-proven lymph node metastasis, as well as those patients with worse pathological tumor features more commonly received $\mathrm{pCT}$. Unfortunately, as the current cohort only included patients undergoing curative-intent hepatic resection for ICC, we were unable to determine the rate of resectability among patients with locally advanced disease. Of note, on final pathology, the use of $\mathrm{PCT}$ did not improve complete $\mathrm{RO}$ resection rates. This is likely multifactorial, but largely be due to the selection of pCT use for patients with tumors characterized by worse pathological features.

Patients who received $\mathrm{pCT}$ had increased minor, but not major perioperative morbidity or mortality rates versus patients who did not receive $\mathrm{PCT}$. This is similar to previously published data regarding the safety of $\mathrm{PCT}$ among patients undergoing resection for intraabdominal cancer. ${ }^{18,19}$ Despite having more advanced disease and undergoing larger hepatic resections, patients who received pCT had equivalent peri-operative mortality and LOS. While long-term OS and DFS were comparable among patients who did and did not receive pCT, propensity score-matched analysis suggested a possible benefit of $\mathrm{pCT}$ regarding both OS and DFS-although the association did not

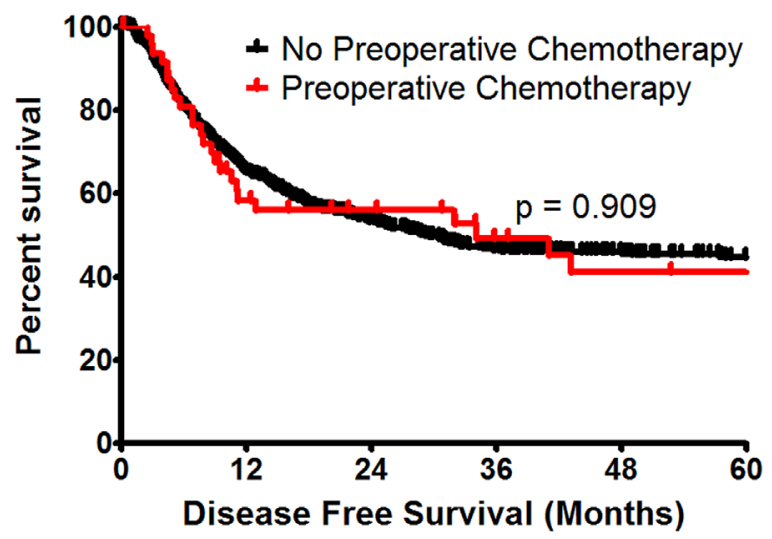

FIGURE 2 Disease free survival stratified by preoperative chemotherapy $(P=0.909)$ 


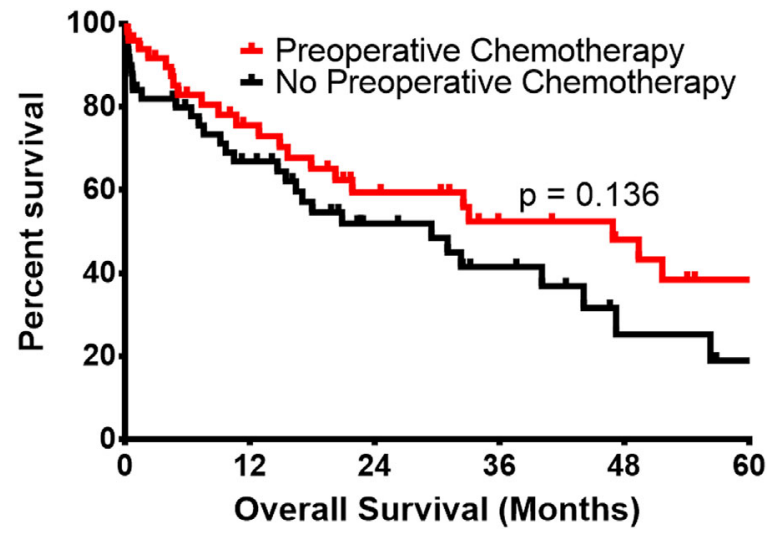

FIGURE 3 Overall survival propensity score-matched patients for resection and microvascular invasion $(P=0.136)$

reach statistical significance. While it is difficult to know, the lack of significance, despite the considerable differences in the point estimates, was likely due to a type II statistical error given the very low utilization of $\mathrm{pCT}$ in the current cohort. Nelson et al had reported that the use of $\mathrm{pCT}$ combined with radiation therapy improved survival outcomes among patients with extrahepatic cholangiogcarcinoma. ${ }^{20}$ In a different study, Tamandl et al reported on 10 patients with ICC who were treated with $\mathrm{pCT}$ and noted no survival benefit. ${ }^{21}$ While the current study was one of the largest series to examine ICC patients to receive pCT $(n=62)$, we similarly failed to find an effect of pCT on long-term outcomes. As noted, however, the sample size was still relatively small and therefore future prospective studies are needed.

In this study, we included 18 patients who received preoperative intra-arterial chemotherapy, as opposed to the 44 patients who received systemic chemotherapy. Intra-arterial therapy consists of the delivery of high doses of chemotherapy directly to the arterial circulation. ${ }^{9}$ This results in high first pass extraction rates and minimizes systemic toxicity, as tumors derive most of their supply from the arterial circulation. ${ }^{9,22}$ The effects of intra-arterial therapy have been described in two clinical trials and a retrospective analysis, which showed promising results in patients with liver-confined disease in a palliative setting. ${ }^{9,23,24}$ In our cohort, the lower percentage of patients with preoperatively confirmed lymph node metastases in the intra-arterial chemotherapy group ( $26.7 \%$ vs $44.7 \%)$, suggests that intra-arterial therapy was most often used preoperatively in patients with suspected borderline resectable disease, as opposed to patients with suspected micrometastatic disease. Although our finding is in line with current literature on patients with irresectable disease, future studies are needed to confirm the validity of this approach prior to a curative resection.

Results of the current study should be interpreted in the context of several limitations. As noted, the number of patients treated with pCT was small as the overall utilization was only $5.9 \%$. Therefore, the lack of statistical significance was likely related to a type II error. Additionally, inherent to all retrospective analyses, there may have been a selection bias regarding the diagnosis and treatment of patients. The inclusion of multiple centers also did not allow for the standardization of operative approach or protocols related to the use of $\mathrm{pCT}$ or adjuvant chemotherapy. The multi-center nature of the study adds to the generalizability of the study, allowing the finding to be applied across a wide range of patient populations.

\section{5 | CONCLUSIONS}

In conclusion, pCT utilization among patients with ICC is higher among patients with more advanced disease. In this large, multi-institutional cohort, the use of $\mathrm{pCT}$ did not impact short-term peri-operative outcomes such as morbidity or LOS. While OS and DFS following resection were not significantly different across treatment groups, propensity matching suggested possible improved outcomes in patients treated with pCT. Further prospective trials are needed, however, to better define the role of pCT and to identify the subset of patients who might yield the most clinical benefit from the use of pCT.

\section{POTENTIAL CONFLICTS OF INTEREST}

Nothing to disclose.

\section{REFERENCES}

1. DeOliveira ML, Cunningham SC, Cameron JL, et al. Cholangiocarcinoma: thirty-one-year experience with 564 patients at a single institution. Ann Surg. 2007;245:755-762.

2. Cillo U, Spolverato G, Vitale A, et al. Liver resection for advanced intrahepatic cholangiocarcinoma: a cost-Utility analysis. World J Surg. 2015;39:2500-2509.

3. Esnaola NF, Meyer JE, Karachristos A, Maranki JL, Camp ER, Denlinger CS. Evaluation and management of intrahepatic and extrahepatic cholangiocarcinoma. Cancer. 2016;122:1349-1369.

4. Olnes MJ, Erlich R. A review and update on cholangiocarcinoma. Oncology. 2004;66:167-179.

5. Darwish Murad S, Kim WR, Harnois DM, et al. Efficacy of neoadjuvant chemoradiation, followed by liver transplantation, for perihilar cholangiocarcinoma at 12 US centers. Gastroenterology. 2012;143:88-98 e3; quiz e14.

6. Nakeeb A, Tran KQ, Black MJ, et al. Improved survival in resected biliary malignancies. Surgery. 2002;132:555-63; discission 63-4.

7. Endo I, Gonen M, Yopp AC, et al. Intrahepatic cholangiocarcinoma: rising frequency, improved survival, and determinants of outcome after resection. Ann Surg. 2008;248:84-96.

8. de Jong MC, Nathan H, Sotiropoulos GC, et al. Intrahepatic cholangiocarcinoma: an international multi-institutional analysis of prognostic factors and lymph node assessment. J Clin Oncol. 2011;29:3140-3145.

9. Konstantinidis IT, Groot Koerkamp B, Do RK, et al. Unresectable intrahepatic cholangiocarcinoma: systemic plus hepatic arterial infusion chemotherapy is associated with longer survival in comparison with systemic chemotherapy alone. Cancer. 2016;122:758-765.

10. Hyder O, Hatzaras I, Sotiropoulos GC, et al. Recurrence after operative management of intrahepatic cholangiocarcinoma. Surgery. 2013;153:811-818.

11. Spolverato G, Yakoob MY, Kim Y, et al. The impact of surgical margin status on long-term outcome after resection for intrahepatic cholangiocarcinoma. Ann Surg Oncol. 2015;22:4020-4028.

12. Wagner A, Wiedmann M, Tannapfel A, et al. Neoadjuvant down-Sizing of hilar cholangiocarcinoma with photodynamic therapy-Long-Term outcome of a phase II pilot study. Int J Mol Sci. 2015;16:26619-26628. 
13. Edge SB, Byrd DR, Compton CC, Fritz AG, Greene FL, Trotti A. AJCC Cancer Staging Manual. 7th ed. Chicago, IL: Springer; 2009.

14. Tran TB, Bal CK, Schaberg K, Longacre TA, Chatrath BS, Poultsides GA. Locally advanced intrahepatic cholangiocarcinoma: complete pathologic response to neoadjuvant chemotherapy followed by left hepatic trisectionectomy and caudate lobectomy. Dig Dis Sci. 2015; 60:3226-3229.

15. Walker EJ, Simko JP, Nakakura EK, Ko AH. A patient with cholangiocarcinoma demonstrating pathologic complete response to chemotherapy: exploring the role of neoadjuvant therapy in biliary tract cancer. J Gastrointest Oncol. 2014;5:E88-E95.

16. Weber SM, Ribero D, O'Reilly EM, Kokudo N, Miyazaki M, Pawlik TM. Intrahepatic cholangiocarcinoma: Expert consensus statement. HPB (Oxford). 2015;17:669-680.

17. Hackert T, Sachsenmaier M, Hinz U, et al. Locally advanced pancreatic cancer: neoadjuvant therapy with folfirinox results in resectability in $60 \%$ of the patients. Ann Surg. 2016;264:457-463.

18. Ejaz A, Spolverato G, Kim Y, et al. Impact of external-Beam radiation therapy on outcomes among patients with resected gastric cancer: a multi-institutional analysis. Ann Surg Oncol. 2014;21:3412-3421.

19. Parikh AA, Gentner B, Wu TT, Curley SA, Ellis LM, Vauthey JN. Perioperative complications in patients undergoing major liver resection with or without neoadjuvant chemotherapy. J Gastrointest Surg. 2003;7:1082-1088.
20. Nelson JW, Ghafoori AP, Willett CG, et al. Concurrent chemoradiotherapy in resected extrahepatic cholangiocarcinoma. Int $J$ Radiat Oncol Biol Phys. 2009;73:148-153.

21. TamandI D, Herberger B, Gruenberger B, Puhalla H, Klinger M, Gruenberger $T$. Influence of hepatic resection margin on recurrence and survival in intrahepatic cholangiocarcinoma. Ann Surg Oncol. 2008;15:2787-2794.

22. Collins JM. Pharmacologic rationale for regional drug delivery. J Clin Oncol. 1984;2:498-504.

23. Jarnagin WR, Schwartz LH, Gultekin DH, et al. Regional chemotherapy for unresectable primary liver cancer: Results of a phase II clinical trial and assessment of DCE-MRI as a biomarker of survival. Ann Oncol. 2009;20:1589-1595.

24. Kemeny NE, Schwartz L, Gonen M, et al. Treating primary liver cancer with hepatic arterial infusion of floxuridine and dexamethasone: does the addition of systemic bevacizumab improve results? Oncology. 2011;80:153-159.

How to cite this article: Buettner S, Koerkamp BG, Ejaz A, et al. The effect of preoperative chemotherapy treatment in surgically treated intrahepatic cholangiocarcinoma patients-A multi-institutional analysis. J Surg Oncol. 2017;115:312-318. https://doi.org/10.1002/jso.24524 